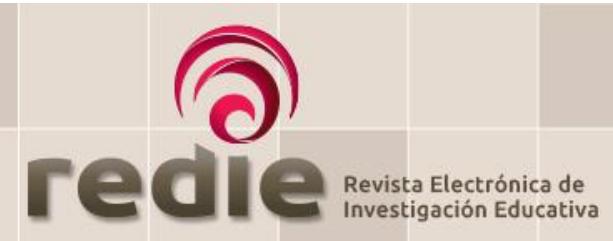

Vol. 22, 2020/e18

\title{
Formación de profesores de Física en Chile: realidad y desafíos ${ }^{1}$
}

\section{Physics Teacher Training in Chile: Reality and Challenges}

Carla Hernández Silva (1) https://orcid.org/0000-0003-2974-4989

Yadrán Gómez-Martínez (2) https://orcid.org/0000-0002-2124-0893

Damián Ruz López (1) https://orcid.org/0000-0003-1812-4565

Mauricio Silva Ugalde (3) https://orcid.org/0000-0003-1040-8707

Silvia Tecpan Flores (1) https://orcid.org/0000-0001-9343-6863

(1) Universidad de Santiago de Chile

(2) Ministerio de Educación de Chile

(3) Universidad de Chile

(Recibido: 18 de octubre de 2018; Aceptado para su publicación: 20 de diciembre de 2018)

Cómo citar: Hernández, C., Gómez-Martínez, Y., Ruz, D., Silva, M. y Tecpan, S. (2020). Formación de profesores de Física en Chile: realidad y desafíos. Revista Electrónica de Investigación Educativa, 22, e18, 1-18. https://doi.ora/10.24320/redie.2020.22.e18.2672

\section{Resumen}

La escasez de profesores de Física en Chile ha constituido un tema de preocupación en los últimos 15 años. El presente trabajo busca identificar y describir las principales características de los actuales programas formativos de pedagogía en Física de universidades pertenecientes al Consejo de Rectores de las Universidades Chilenas (CRUCH). La investigación es descriptiva, ex post facto, con diseño de análisis histórico de datos y técnicas de análisis cualitativo. Entre los resultados destaca la centralización geográfica y las brechas de género en cuanto a matrícula y titulación. Desde lo curricular, las asignaturas del área de Física y de Educación aparecen en los programas como líneas paralelas y en porcentajes muy similares, lo que sugiere una disociación entre ambas áreas. Se discuten las implicancias para programas de formación inicial docente y se proponen líneas de acción para futuras investigaciones basadas en los hallazgos.

Palabras clave: Formación de docentes, enseñanza de la Física, planes educativos.

\section{Abstract}

The lack of physics teachers in Chile has been a cause for concern for the last 15 years. This study aims to identify and describe the key features of current physics teacher training programs in universities in the Council of Rectors of Chilean Universities (CRUCH). This descriptive and ex post facto research employs historical data analysis and qualitative analysis techniques. The main findings include a geographic centralization and gender gaps in enrollment and graduation rates. The physics and education courses appear to run parallel and feature in similar proportions, suggesting a dissociation between the two areas. The implications for teacher training programs are discussed and lines of action for future research are proposed based on the findings.

Keywords: Teacher education, physics education, educational planning.

\footnotetext{
${ }^{1}$ Este estudio fue realizado gracias al apoyo de CONICYT a través del proyecto FONDECYT número 11170580.
} 


\section{Introducción}

Durante los últimos años se han implementado en Chile diversas iniciativas para fortalecer el desarrollo profesional docente. Entre ellas, se han establecido incentivos como la Beca Vocación de Profesor ${ }^{2}$ para motivar a los jóvenes que obtienen altos puntajes en la Prueba de Selección Universitaria (PSU) a cursar carreras de pedagogía financiando el estado chileno sus estudios. Por otra parte, se apunta también a generar un sistema de acompañamiento para los primeros años de ejercicio docente y diagnosticar de manera temprana la calidad de la formación recibida a través de un sistema de Evaluación Nacional Diagnóstica.

Junto con lo anterior, el Ministerio de Educación de Chile (Mineduc) promovió en 2008 la elaboración de estándares orientadores para carreras de pedagogía con el objetivo de guiar los procesos formativos en las universidades que imparten este tipo de programas (Mineduc, 2012). Dichos estándares contemplan directrices sobre lo que un profesor debe saber y saber hacer desde el punto de vista disciplinar y pedagógico (Sotomayor y Gysling, 2011); no obstante, representan sólo un referente que las universidades pueden incorporar, o no, en su oferta formativa. Cabe destacar que no es objetivo de los estándares orientadores proponer una estructura única y definida para articular las mallas curriculares de las carreras de pedagogía a nivel nacional, por lo tanto, cada institución de educación superior mantiene su autonomía para estructurarlos.

Frente a estas políticas y lógicas organizacionales se esperaría disponer de una línea base para contribuir desde la investigación en el área a la formación de profesores de Física; sin embargo, aun cuando se dispone de estudios que permiten caracterizar en términos generales la Formación Inicial Docente (FID) en Ciencias en Chile (Arellano et al., 2008; Cofré et al., 2010), actualmente no se cuenta con evidencias suficientes y sistematizadas que permitan visualizar un panorama específico para el caso de Pedagogía en Física, sobre todo después de que se detectara la escasez de docentes en el área hace 15 años (Claro, 2003).

En este contexto, el presente artículo realiza una revisión sobre el estado actual de la formación docente de Física en Chile, con el objetivo de identificar y describir las características de los programas formativos vigentes aportando evidencias a la reflexión y discusión sobre nuevos diseños curriculares.

La formación inicial de profesores es uno de los temas principales de la agenda política de los países que se preocupan por mejorar la calidad y equidad en educación (Barber y Mourshed, 2008). A nivel internacional, la formación docente se realiza con diferentes modelos y sistemas de pruebas habilitantes (Montecinos y Walker, 2018; Vaillant, 2013). En el contexto chileno, para ser docente se deben cursar estudios en alguna universidad, estatal o privada, las cuales otorgan el grado académico de licenciado en Educación junto al título de profesor con especialidad, dependiendo de la disciplina (Ávalos, 2003). Sin embargo, no existe consenso respecto a si la formación de docentes debe estar a cargo del área disciplinar o pedagógica dentro de cada institución, o si se requiere de una nueva organización que favorezca la integración entre ambas áreas. Al respecto, persisten tensiones de naturaleza ideológica sobre si un docente debe tener mayor formación académica de Física o de Pedagogía al término de su carrera (Justino y de Almeida, 2016).

En un estudio realizado por la Unesco (Unesco y Orealc, 2012) sobre las tendencias en educación superior en Latinoamérica, se reconoce el desequilibrio existente entre la formación disciplinar y pedagógica dentro de los programas de formación docente en general. Esta situación podría representar un factor que tiende a dificultar un desarrollo efectivo del conocimiento pedagógico del contenido para el futuro profesor (Shulman, 2015), dado que el conocimiento disciplinar se estaría abordando en asignaturas separadas y desarticuladas con las didácticas específicas. Además, existen evidencias que han apuntado a la baja complementariedad entre formación teórica y práctica, y a debilidades en aspectos metodológicos de la enseñanza (Gatti, 2015).

\footnotetext{
${ }^{2}$ http://portal.beneficiosestudiantiles.cl/becas-y-creditos/beca-vocacion-de-profesor-pedagogias-bvp-pedagogia
} 
Al respecto, un artículo publicado por López et al. (2000) concluye que la importancia de integrar los aspectos disciplinares con los pedagógicos radica en cómo las ideas sobre ciencia y aprendizaje del docente influyen en su desempeño en el aula. Así, cobra relevancia la necesidad de que el docente tenga conocimiento de la disciplina, pero al mismo tiempo posea herramientas que le permitan convertir el conocimiento científico en un conocimiento enseñable (Chevallard, 2014), herramientas que no pueden aprenderse de forma adecuada en cursos de pedagogía aislados de la disciplina.

En este contexto, es necesaria la incorporación de asignaturas de didáctica de la Física en los programas de las carreras, que brinden instancias para que el futuro docente reflexione sobre su conocimiento disciplinar y lo utilice para transformarlo en un conocimiento enseñable atendiendo a las características del contexto escolar. En algunos estudios se ha encontrado evidencia de que los estudiantes de Pedagogía en Física enfrentan los cursos de didáctica con dificultades en su conocimiento disciplinar (Fagúndez y Castells, 2012; Hernández et al., 2018), generando así otro punto de tensión.

En Chile, los resultados de la prueba diagnóstica realizada a los estudiantes de últimos semestres de carrera en el marco de la Evaluación Nacional Docente han evidenciado algunas carencias en la formación disciplinar de los futuros profesores de Física, basándose en los bajos porcentajes de logro en la media nacional (Centro de Perfeccionamiento, Experimentación e Investigaciones Pedagógicas [CPEIP], 2018). Si bien no es objetivo de este artículo abordar las características de la evaluación ni cuestionar su pertinencia, los resultados ponen de manifiesto la necesidad de mejorar la formación de profesores no sólo desde el punto de vista de la disciplina, sino también de las oportunidades que los futuros docentes tienen a lo largo de la carrera para cuestionar sus propios aprendizajes y mejorarlos. Desarrollar este aspecto es posible incorporando la didáctica específica como una línea formativa transversal asociada a la enseñanza y aprendizaje de la disciplina, y no sólo como una asignatura adicional y aislada en la malla curricular (Bolívar, 2005).

Aunque existe preocupación respecto a los resultados del diagnóstico de egreso, cabe poner atención también al perfil de estudiantes que ingresan a las carreras de pedagogía. A nivel internacional se observa que los países de la OCDE que obtienen mejores resultados en evaluaciones estandarizadas son los que han establecido mayores exigencias para ingresar a carreras de formación docente (OCDE, 2016). En estos programas los estudiantes suelen estar entre el 30\% de mejor desempeño en cada cohorte anual de egresados de la educación secundaria (Claro et al., 2013). En comparación, los postulantes a las carreras de pedagogía en Chile provienen, en su mayoría, del 50\% con menor puntaje en la PSU (Cabezas y Claro, 2011), lo que coincide con la situación a nivel latinoamérica (Unesco y Orealc, 2012).

Con la entrada en vigor del nuevo Sistema de Desarrollo Profesional Docente en Chile a partir de 2017, el ingreso a las carreras de pedagogía requiere que el postulante obtenga al menos 500 puntos (de los 850 posibles) en la PSU, en las pruebas obligatorias de Matemática y Lenguaje, o estar dentro del $30 \%$ de estudiantes con mayor ranking de notas de educación secundaria (en el establecimiento donde cursó sus estudios). Además, se espera que en el año 2023 el puntaje mínimo PSU requerido aumente a 550 puntos o que los estudiantes pertenezcan al $10 \%$ de estudiantes con mejor ranking de notas. Sin embargo, estas medidas están aún distantes de la experiencia internacional y generan otras tensiones entre quienes cuestionan que el ingreso a la universidad sea mediante procesos selectivos, y no como un derecho al que cualquier estudiante pueda optar.

Particularmente en el caso de Ciencias, los resultados PSU de los estudiantes también reflejan debilidades en la formación científica escolar a nivel de conocimientos y habilidades, coherentes con resultados informados por otras evaluaciones estandarizadas como PISA o SIMCE (Agencia Calidad de la Educación, 2014; 2018). Estas debilidades constituyen un desafío para nuevas generaciones de profesores que requieren adquirir herramientas durante su formación inicial, para luego emplearlas en el ejercicio docente. Al respecto, con la finalidad de fortalecer el proceso de alfabetización científica en la escuela, investigadores coinciden en la importancia de formar profesores con una visión crítica y naturalizada de las ciencias (Adúriz-Bravo, 2005; Matthews, 2014; Quintanilla, 2017), y de la Física en particular (Koponen y Mantyla, 2006; Sasseron et al., 2013). Es decir, un profesor de Física no sólo debe conocer el contenido que está enseñando sino también las motivaciones y problemas que originaron el conocimiento científico y cuáles fueron las dificultades y obstáculos epistemológicos involucrados; así como estar al tanto de los 
desarrollos recientes, sus perspectivas y límites de validez (Carvalho y Gil, 2011; Gómez et al., 2014).

Considerando los aspectos de contexto y perspectivas teóricas mencionadas, se presenta a continuación el proceso y resultado del análisis realizado para las carreras de Pedagogía en Física en Chile.

\section{Método}

En coherencia con la naturaleza del objetivo propuesto, el estudio se desarrolló con un enfoque exploratorio y descriptivo, desde una mirada macroscópica a nivel nacional sobre el estado de la FID en Física. La investigación es descriptiva, ex post facto (Hernández et al., 2013) con diseño de análisis histórico de datos (Vogt, 2013), dado que se consultaron las bases de datos existentes, de dominio público, y la información dispuesta por cada institución de educación superior estudiada. Por último, se utilizaron técnicas de análisis de datos cualitativos (Hernández et al., 2013).

Para caracterizar la FID en Física de las universidades pertenecientes al CRUCH se siguieron las siguientes etapas: 1) Identificación de la oferta académica para carreras de Pedagogía en Física de todas las universidades del $\mathrm{CRUCH}$; 2) Análisis de programas/mallas curriculares de las carreras identificadas, en función de las asignaturas por área de conocimiento; 3) Análisis del perfil académico de ingreso; 4) Determinación de la cantidad de estudiantes matriculados en primer año, entre 2005 y 2017; 5) Determinación de la cantidaad de estudiantes titulados por año, entre 2007 y 2016; 6) Análisis de contenido del perfil de egreso.

Cabe mencionar que, dado que la información en las fuentes de consulta es dinámica, se estableció como fecha de corte el mes de mayo de 2018. Los análisis se realizaron en función de la información disponible a la fecha indicada, por lo que es posible que existan actualizaciones posteriores que no estén consideradas en este estudio.

Para el cumplimiento de las etapas 1, 2 y 4 se consultó la información publicada en el sitio oficial de cada institución del CRUCH durante el Proceso de Admisión 2018, determinando así la oferta académica de las universidades que imparten la carrera de Pedagogía en Física a nivel nacional. Del mismo modo, se consultó la estructura curricular para cada carrera identificada.

Para las etapas 1, 2, 3 y 6 se diseñaron rejillas de análisis (Giroux y Tremblay, 2004) que permitieron sistematizar la información recabada. Específica y complementariamente, los análisis de las etapas 3, 4 y 5 se realizaron con Microsoft Excel.

Para las etapas 3 y 4 se utilizó la base de datos del Consejo Nacional de Educación (CNED, 2017) llamada "INDICES_Matricula_Periodo_2005_2017". De esta base también se extrajo información referente al perfil académico de ingreso de los estudiantes. Para determinar la cantidad de titulados de las mismas carreras identificadas, se utilizó la base de datos del Servicio de Información de Educación Superior (SIES, 2017).

En relación con la etapa 6, los perfiles de egreso de todas las carreras fueron analizados por contenido utilizando el software Atlas.ti. El análisis se llevó a cabo a través del siguiente proceso: a) Identificación de categorías emergentes a partir de la lectura de los distintos perfiles, para codificar el texto; b) Organización y contraste de las categorías establecidas con lo señalado por la literatura; y c) Análisis de los fragmentos codificados para determinar el grado de presencia de las categorías. Para la validación de los resultados cualitativos en esta etapa se recurrió a la triangulación entre pares (Creswell y Plano-Clark, 2011).

A continuación se presentan y fundamentan las categorías utilizadas para la codificación del perfil de egreso en la etapa VI, basadas en la literatura disponible. 
Tabla I. Categorías y definiciones utilizadas en la codificación del análisis de los perfiles de egreso

\begin{tabular}{|c|c|}
\hline Categoría & $\begin{array}{l}\text { Definición } \\
\text { Se identifican en cada categoría todas aquellas descripciones en el perfil de egreso que } \\
\text { hacen referencia al: }\end{array}$ \\
\hline Uso de tIC & $\begin{array}{l}\text { Uso y gestión de las nuevas tecnologías de la información y la comunicación, desde el } \\
\text { uso de software hasta la búsqueda y gestión de información en Internet (Tejada, } \\
\text { 2009). }\end{array}$ \\
\hline $\begin{array}{l}\text { Conocimiento } \\
\text { disciplinar }\end{array}$ & $\begin{array}{l}\text { Dominio por parte del futuro docente del conocimiento específico de la disciplina } \\
\text { (Tejada, 2009). }\end{array}$ \\
\hline $\begin{array}{l}\text { Conocimiento } \\
\text { pedagógico }\end{array}$ & $\begin{array}{l}\text { Dominio de todos aquellos procesos y prácticas de enseñanza y aprendizaje que } \\
\text { tributan al logro de objetivos de aprendizaje (Harris et al., 2009). }\end{array}$ \\
\hline $\begin{array}{l}\text { Demuestra } \\
\text { competencias }\end{array}$ & Competencias docentes referidas al ámbito metodológico y social (Tejada, 2009). \\
\hline Profesional reflexivo & $\begin{array}{l}\text { Continua práctica reflexiva que realizará el futuro docente en torno a su ejercicio } \\
\text { (Tirado, 2015). }\end{array}$ \\
\hline Docente investigador & $\begin{array}{l}\text { Actividad de los futuros docentes como investigadores y generadores de teorías a } \\
\text { partir del análisis y reflexión de la propia práctica (Garcés, 2018). }\end{array}$ \\
\hline $\begin{array}{l}\text { Prácticas tempranas } \\
\text { y profesionales }\end{array}$ & $\begin{array}{l}\text { Incorporación de instancias de práctica a lo largo de su formación, como espacios que } \\
\text { permitan concretar las aspiraciones y finalidades del currículum de la carrera (Escobar, } \\
\text { 2007). }\end{array}$ \\
\hline $\begin{array}{l}\text { Ética y compromiso } \\
\text { social }\end{array}$ & $\begin{array}{l}\text { Competencias genéricas, refiriéndose a la capacidad del futuro docente de mostrar en } \\
\text { su desempeño una actitud ética, comprometida y responsable socialmente frente a } \\
\text { contextos diversos (Maura y Tirados, 2008). }\end{array}$ \\
\hline $\begin{array}{l}\text { Liderazgo y trabajo } \\
\text { en equipo }\end{array}$ & $\begin{array}{l}\text { Capacidad de los futuros docentes de organizar y gestionar grupos de trabajo, así } \\
\text { como adoptar el rol de líder en la dirección de reuniones o espacios de trabajo } \\
\text { (Perrenoud, 2004). }\end{array}$ \\
\hline
\end{tabular}

Los resultados de la codificación de los perfiles fueron posteriormente analizados por contenido y frecuencia utilizando Microsoft Excel.

\section{Resultados y discusión}

La presentación de resultados se realiza siguiendo el orden de las etapas planteadas en la metodología y guarda relación con los aspectos considerados de interés en la formación de profesores de Física.

\subsection{Identificación de la oferta académica para Pedagogía en Física en las universidades del CRUCH (Etapa 1)}

En Chile, la carrera de Pedagogía en Física se imparte a nivel de pregrado en 14 Universidades del país, otorgando una licenciatura como grado académico junto al título profesional de profesor (Ávalos, 2003). Dentro de la oferta académica actual, algunas instituciones ofrecen el título de profesor de Física compartido junto con Matemática o como una mención dentro del programa de Pedagogía en Ciencias. En la tabla II se presentan en detalle cada uno de los programas de Pedagogía en Física existentes actualmente. 
Tabla II. Descripción general de la oferta formativa de Pedagogía en Física a nivel nacional

\begin{tabular}{|c|c|c|c|}
\hline Administración & Institución & Programa & Facultad que lo imparte \\
\hline \multirow{10}{*}{ Estatal } & Universidad de Тагарасá & Física y Matemática & Ciencias \\
\hline & Universidad Arturo Prat & Matemática y Física & Ciencias Humanas \\
\hline & Universidad de La Serena & Matemáticas y Física & Ciencias \\
\hline & Universidad de Playa Ancha & Física & Ciencias Naturales y Exactas \\
\hline & Universidad de Chile & $\begin{array}{l}\text { Educación Media } \\
\text { en Matemática y Física }\end{array}$ & $\begin{array}{l}\text { Ciencias (en convenio con la } \\
\text { Facultad de Filosofía y } \\
\text { Humanidades) }\end{array}$ \\
\hline & $\begin{array}{l}\text { Universidad de Santiago } \\
\text { de Chile }\end{array}$ & Física y Matemática & Ciencia \\
\hline & $\begin{array}{l}\text { Universidad Metropolitana } \\
\text { de Ciencias de la Educación }\end{array}$ & Física & Ciencias Básicas \\
\hline & Universidad de Talca & $\begin{array}{l}\text { Educación Media } \\
\text { en Matemática y Física }\end{array}$ & Ciencias de la Educación \\
\hline & Universidad del Bío-Bío & $\begin{array}{l}\text { Ciencias Naturales con } \\
\text { mención en Física }\end{array}$ & Educación y Humanidades \\
\hline & Universidad de La Frontera & Ciencias mención Física & $\begin{array}{l}\text { Educación, Ciencias Sociales y } \\
\text { Humanidades }\end{array}$ \\
\hline \multirow{4}{*}{ Privada } & $\begin{array}{l}\text { Pontificia Universidad } \\
\text { Católica de Chile }\end{array}$ & Física & Educación \\
\hline & $\begin{array}{l}\text { Pontificia Universidad } \\
\text { Católica de Valparaíso }\end{array}$ & Física & Ciencias Básicas \\
\hline & $\begin{array}{l}\text { Universidad Católica del } \\
\text { Maule }\end{array}$ & $\begin{array}{l}\text { Ciencias con mención } \\
\text { en Física }\end{array}$ & Ciencias Básicas \\
\hline & Universidad de Concepción & Ciencias Naturales y Física & Educación \\
\hline
\end{tabular}

Al observar la distribución por regiones se identificó que el 36\% de los programas de Pedagogía en Física se concentra en la zona centro del país (figura 1) y que un $71 \%$ de ellos se imparte en Instituciones de Educación Superior ( IES) públicas.

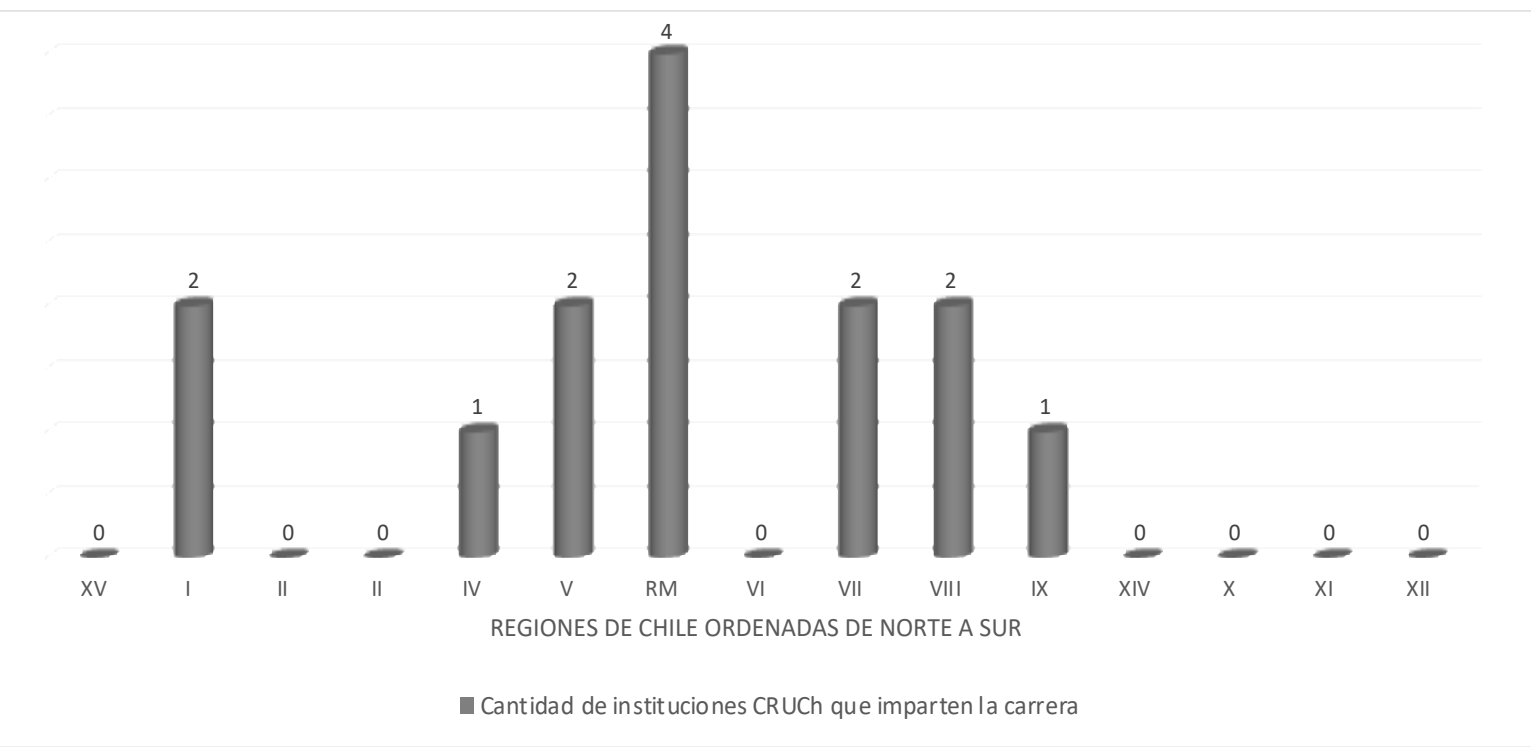

Figura 1. Distribución geográfica de programas de Pedagogía en Física impartidos por región 
En las 14 instituciones identificadas se observa que la carrera de Pedagogía en Física se imparte casi de manera equitativa entre facultades de ciencias básicas (43\%), también llamadas en algunos casos ciencias naturales o ciencias exactas, y en las facultades de humanidades, también llamadas de educación, ciencias sociales o ciencias humanas (57\%).

3.2 Análisis de programas formativos en función del número de asignaturas por área de conocimiento y prácticas pedagógicas (Etapa 2)

Las carreras de Pedagogía en Física identificadas tienen una duración de nueve o diez semestres. A partir de la información disponible para cada institución, se contabilizaron las asignaturas que se imparten por área de conocimiento. La proporción de asignaturas que cada programa formativo incluye se distinguió de acuerdo con las siguientes áreas: Física, Educación y Psicología, Didáctica (generales o específicas), Naturaleza de las Ciencias, Matemática, Prácticas Pedagógicas e Investigación Educativa. En cada área se han considerado también asignaturas llamadas "Taller de...". Además, cabe señalar que no se incluyeron en este análisis las asignaturas de formación complementaria, por no ser de carácter permanente.

Dado que no es objetivo del estudio establecer una comparación entre instituciones, la figura 2 resume la distribución de asignaturas por área de conocimiento para cada Universidad (U) numerada de forma aleatoria. El 100\% para cada institución es el número total de asignaturas que incluye cada programa formativo en su propia malla.

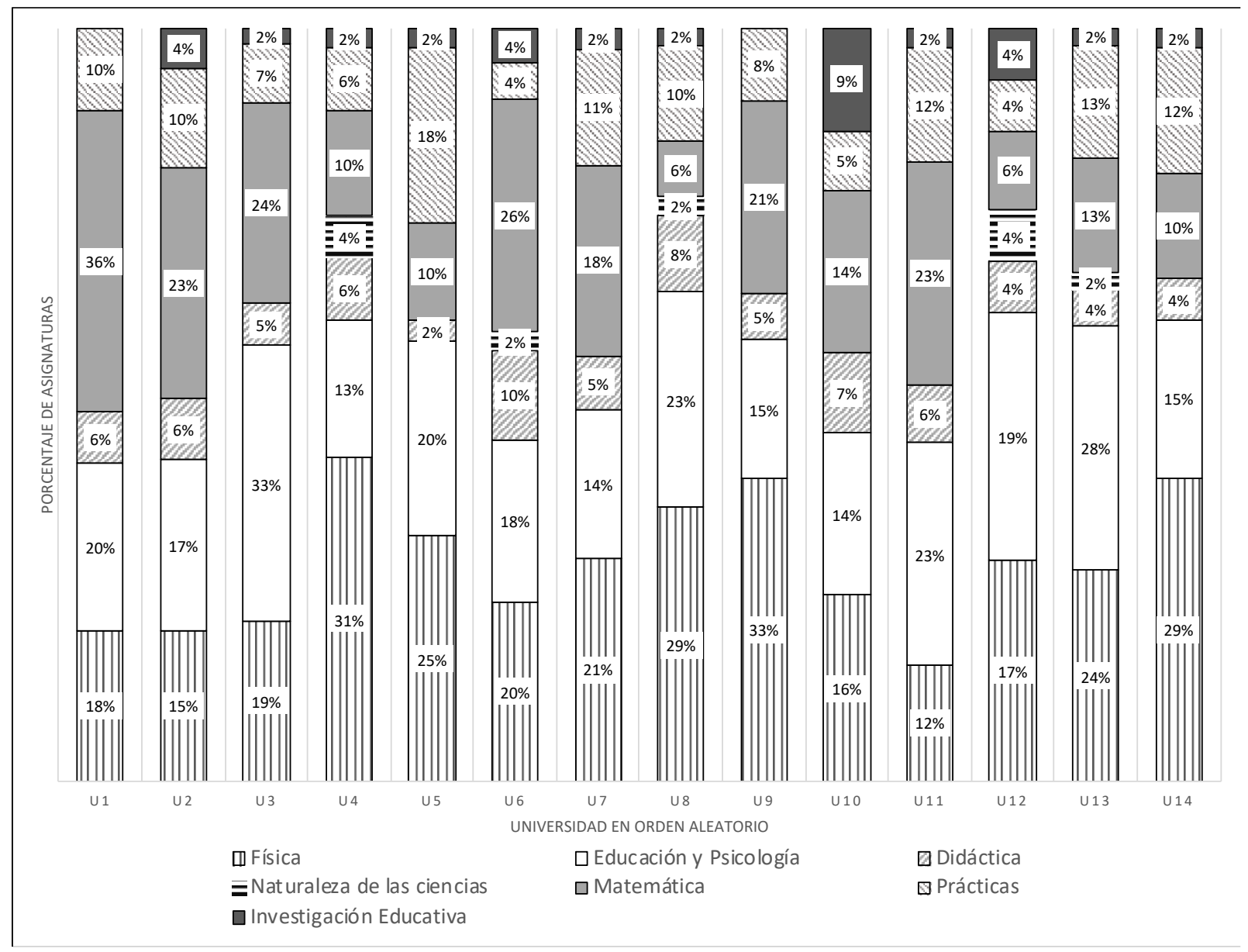

Fuente: Elaboración propia.

Figura 2. Distribución de asignaturas por área de conocimiento en el programa de cada universidad 
A partir de los resultados es posible observar que todos los programas formativos tienen cursos de práctica pedagógica y de didáctica, estos últimos en una baja proporción, lo que resulta preocupante por la importancia que el área tiene para la formación profesional específica en la disciplina (Atalar y Ergun, 2018). Del mismo modo, se observa una baja presencia de asignaturas de investigación educativa, las cuales son esenciales para disminuir brechas entre el mundo académico y el contexto escolar (Roth, 2007). Al respecto, se considera esencial que los futuros docentes logren identificar los problemas reales que enfrentan en el contexto escolar, pudiendo abordarlos con base en la investigación existente referenciada a su propio contexto.

Se evidencia también la compleja situación de que sólo 5 de los 14 programas formativos incorporan asignaturas referidas a naturaleza de las ciencias, y en ellos el porcentaje oscila entre $2 \%$ y $4 \%$. En este análisis se han incluido, agrupadas bajo este concepto, las asignaturas de Historia de la Física, Epistemología o Filosofía de la ciencia. Diversos autores coinciden en la importancia de la formación metateórica de los profesores de ciencias, que influye en las propias concepciones acerca de la naturaleza de la ciencia (Cuellar y Siso, 2017; Quintanilla et al., 2005).

Por otra parte, se observa que las asignaturas de Física representan desde un 12\% hasta un 33\% en los distintos programas de FID en Física, muy similar al porcentaje de representatividad de asignaturas de Educación y Psicología.

En la figura 3 se compara la cantidad de asignaturas de Física y de Pedagogía (área Educación y Psicología), dependiendo de si la facultad que acoge la carrera es de Ciencias (Básicas/Naturales/Exactas) o de Humanidades (Educación/Ciencias Humanas/Ciencias Sociales).

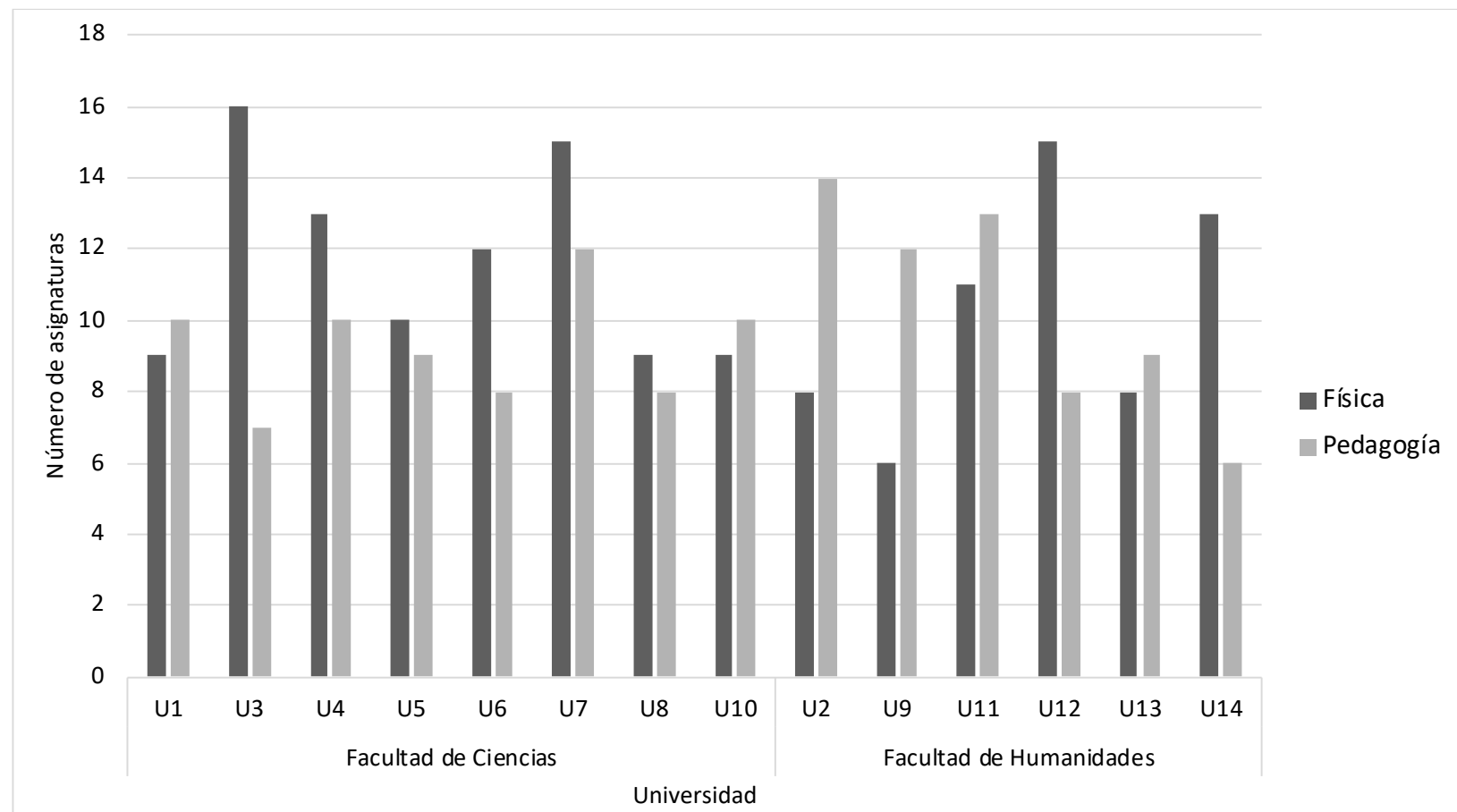

Fuente: Elaboración propia.

Figura 3. Distribución de asignaturas de Física y Pedagogía por tipo de facultad que imparte la carrera

A partir de estos datos se logra distinguir una predominancia de asignaturas disciplinares en un mayor número de carreras que pertenecen a facultades de Ciencia y predominancia de asignaturas pedagógicas en un mayor número de carreras que pertenecen a facultades de Humanidades. 


\subsection{Análisis del perfil académico de ingreso (Etapa 3)}

Respecto del número de vacantes disponibles para acceder a la carrera de Pedagogía en Física a nivel nacional, se observa un leve incremento en los cupos totales disponibles en los últimos dos años en la figura 4.

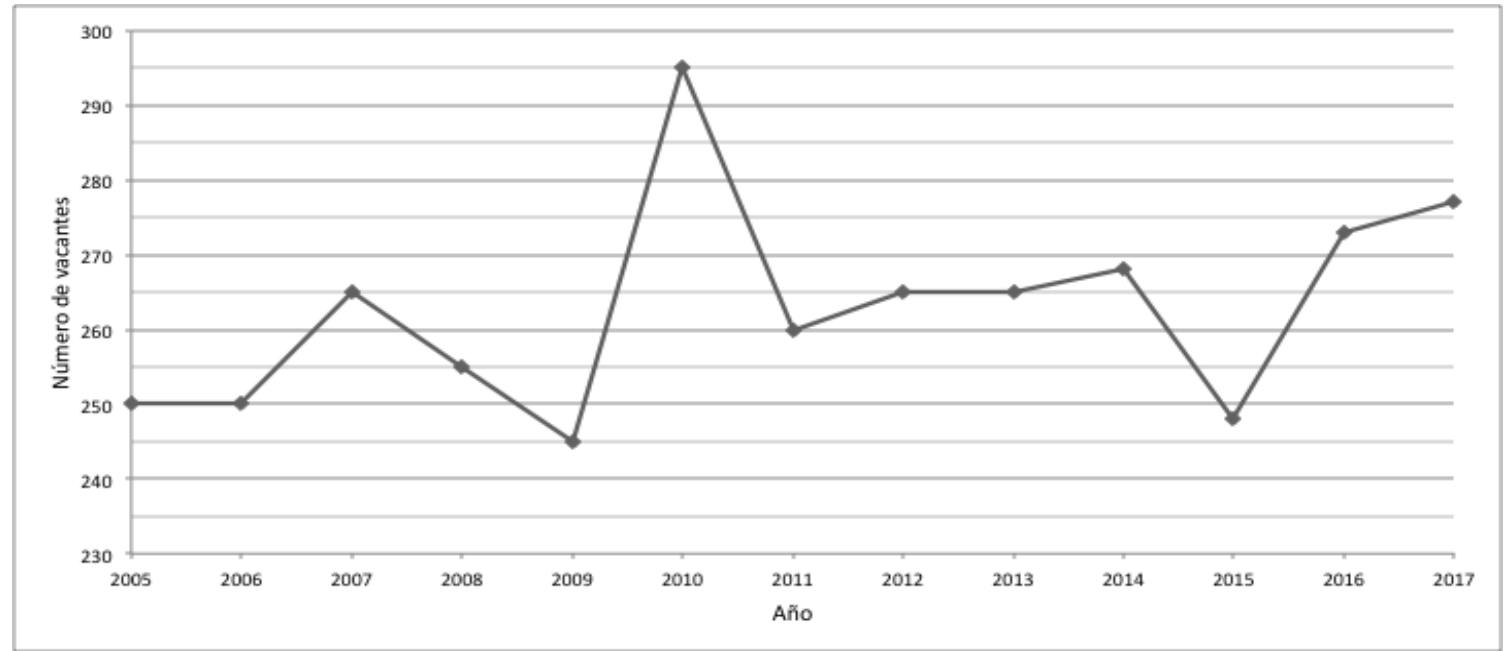

Fuente: Elaboración propia a partir de CNED (2017).

Figura 4. Cantidad de vacantes para ingresar a la carrera de Pedagogía en Física a nivel nacional

Las fluctuaciones se deben al aumento o disminución en el número de vacantes que cada universidad informó en la base de datos del CNED. Históricamente algunas instituciones han aumentado sus cupos mientras que otras los han disminuido drásticamente, por lo que a pesar de que nuevas carreras se abrieron en los últimos dos años el número total de vacantes no tiene un impacto significativo respecto a la tendencia general histórica.

En cuanto al perfil de estudiantes que ingresa a la carrera, se observa un aumento en el puntaje PSU promedio (que considera puntaje de Lenguaje y Matemática) a partir del año 2011.

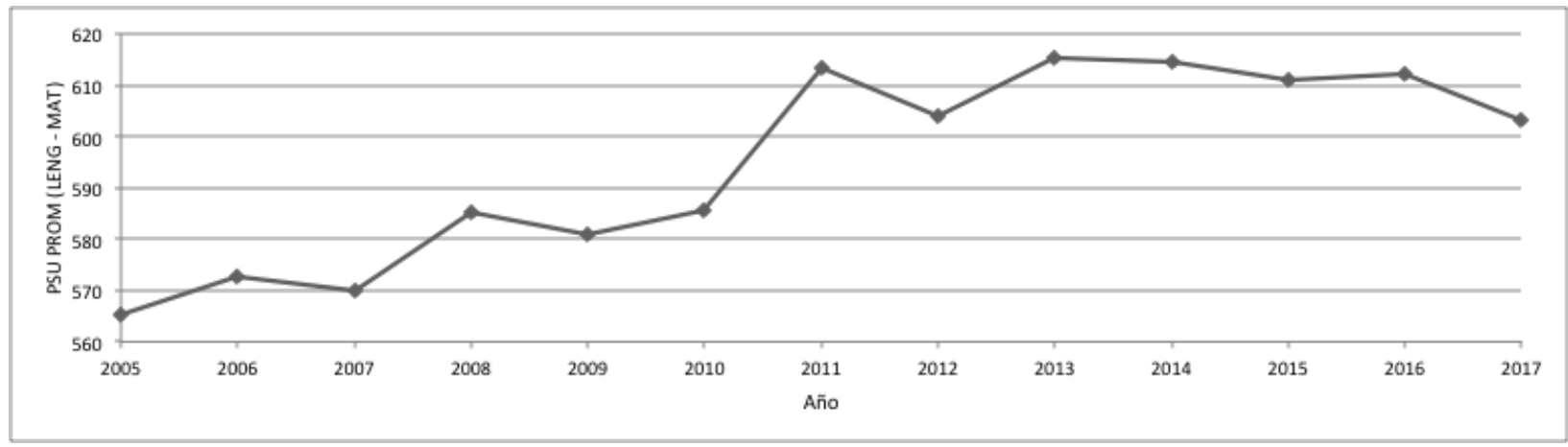

Fuente: Elaboración propia a partir de CNED (2017).

Figura 5. Puntajes pSu promedio de estudiantes que ingresaron a Pedagogía en Física a nivel nacional, en primer año

Si bien el aumento en el puntaje PSU promedio en los últimos años podría relacionarse con la puesta en vigencia de la Beca Vocación de Profesor, éste no parece ser un factor que influya en el número total de estudiantes que optan por estudiar Pedagogía en Física, pues el número de vacantes depende de cada institución y no ha aumentado significativamente, a pesar de la existencia de la beca. En este sentido, algunos estudios han revelado que dicha beca ha contribuido a elevar el puntaje de admisión en las 
carreras de educación, en general, disminuyendo la brecha con otras carreras universitarias (Claro et al., 2013) como ocurre en este caso con Pedagogía en Física.

Para complementar el perfil de ingreso cabe mencionar que los estudiantes que se matriculan en Pedagogía en Física provienen de un rango de Notas de Enseñanza Media (NEM ${ }^{3}$ ) promedio, cercano a un 6.0 (la calificación máxima es de 7.0 en el sistema escolar), lo que en equivalencia con la escala de puntaje PSU es ligeramente mayor, como muestra la figura 6.

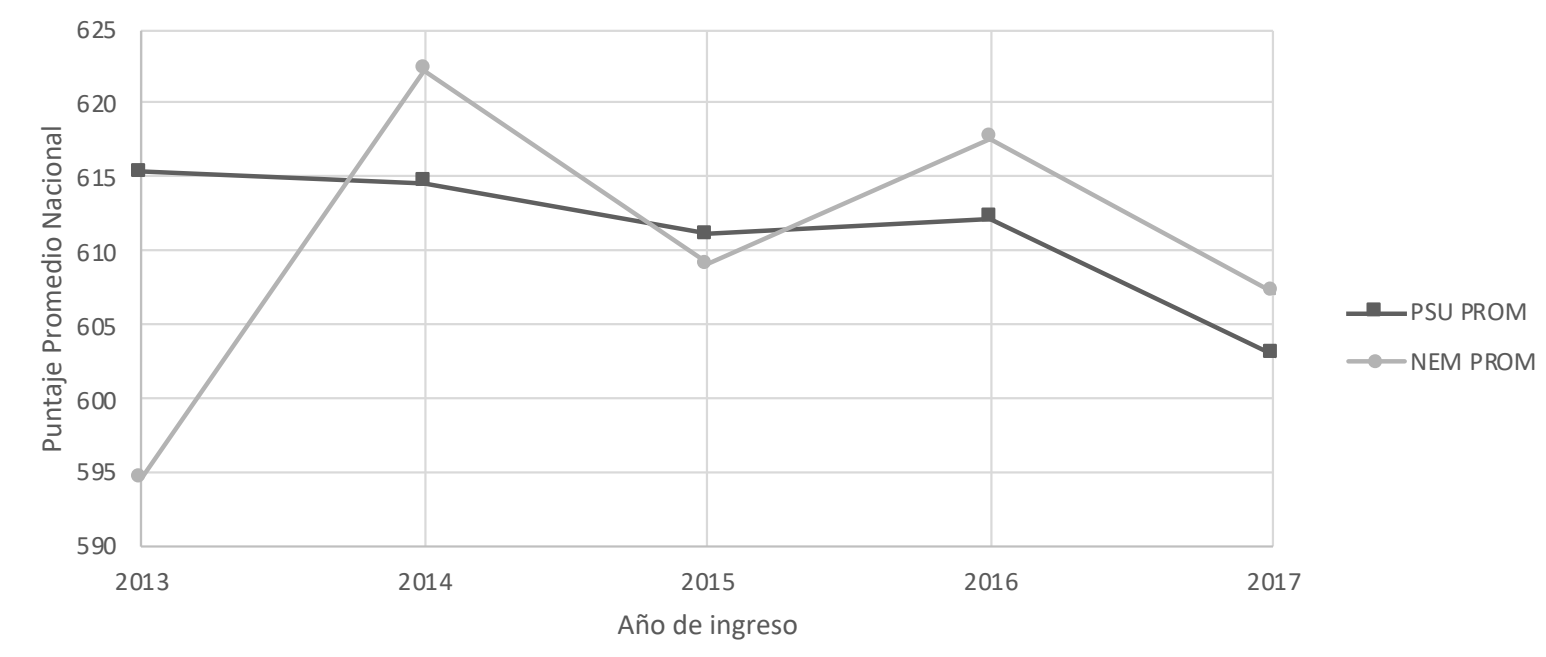

Fuente: Elaboración propia a partir de CNED (2017).

Figura 6. Comparación de puntajes PSU y NEM de estudiantes que ingresan a Pedagogía en Física, a nivel nacional

\subsection{Determinación de la cantidad de matriculados por año, en primer año (Etapa 4)}

Según las tasas de matrícula en el período 2007-2017, en Chile ingresó a estudiar una carrera de Pedagogía en Física un promedio de 259 estudiantes por año (figura 7), lo que corresponde a un 3.2\% de la cantidad de matriculados que ingresó a estudiar una carrera de pedagogía para enseñanza secundaria en 2017 (Centro de Investigación Avanzada en Educación [CIAE], 2017). En la obtención de esta cifra se desestimó el ingreso de estudiantes a carreras de pedagogía en ciencias con mención en Física, Química o Biología, ya que no es posible distinguir en la base de datos utilizada cuál fue la mención elegida dentro del plan de estudio.

Por otra parte, al distinguir por género, es posible observar que históricamente a nivel nacional ha sido mayor la cantidad de hombres matriculados que mujeres. La tasa de matrícula se ha calculado dividiendo el número de matriculados por el total nacional en cada año, para primer año.

${ }^{3}$ http://www.psu.demre.cl/proceso-admision/factores-seleccion/notas-ensenanza-media 


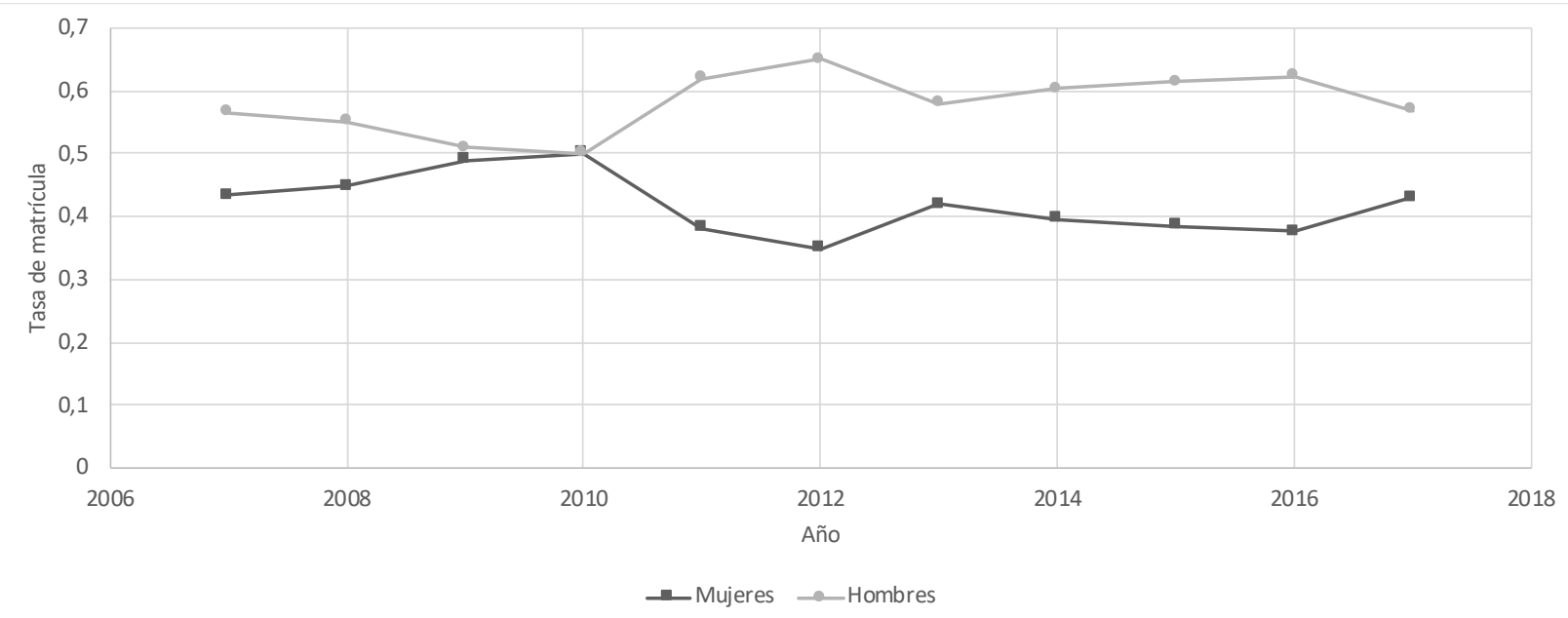

Fuente: Elaboración Propia a partir de CNED (2017).

Figura 7. Tasa de matrícula de estudiantes que ingresaron a Pedagogía en Física a nivel nacional entre 2007 y 2017

Si bien las carreras de pedagogía suelen tener mayor porcentaje de estudiantes mujeres en Chile (Ávalos, 2003), se observa que en el caso de Física, como disciplina, la carrera de formación docente tiene predominio de estudiantes hombres, tanto en la tasa de matrícula como en la de titulados. Sólo un $42 \%$ de los estudiantes que ingresaron a estudiar la carrera en el período 2007-2017 son mujeres, lo que contrasta con el índice a nivel nacional donde, en 2017, un 74\% de los estudiantes matriculados para estudiar carreras en el área de Educación, son mujeres (CNED, 2017).

\subsection{Determinación de la cantidad de titulados por año (Etapa 5)}

En el caso de los estudiantes titulados de la carrera a nivel nacional se observa que un promedio de 215 estudiantes por año obtuvo su título de profesor de Física en Chile durante el período 2007-2016 (figura 8), una cifra baja si se tiene presente la tasa de retiro de profesores en la disciplina (CIAE, 2018). Al igual que en la etapa 4, no se han considerado en estas cifras los egresados de carreras de pedagogía en ciencia por desconocer la cantidad exacta que optó por la mención en Física.

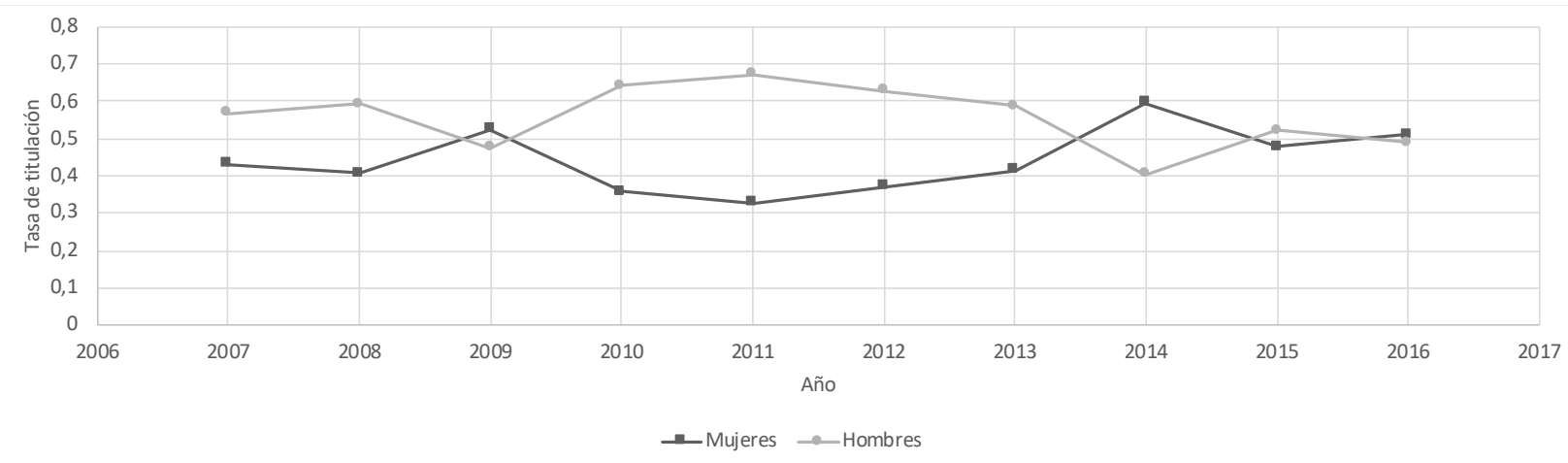

Fuente: Elaboración propia a partir de base publicada en www.mifuturo.cl (SIES, 2017).

Figura 8. Tasa de titulación de estudiantes que ingresaron a Pedagogía en Física a nivel nacional entre 2007 y 2016 
La relación entre titulados y matriculados (ver figura 9) se define como el cociente entre dichos valores para un determinado año académico, y se considera un indicador de interés en la salida del sistema universitario (Bará et al., 2002). Para las carreras de Pedagogía en Física, la relación promedio de los últimos 10 años ha sido de 0.86. Sin embargo, cabe destacar que por las características de las bases de datos utilizadas no es posible determinar la tasa de retención de las carreras, pues se requiere disponer de mayor información sobre la trayectoria académica de cada estudiante, por lo que es un valor referencial que debe ser tomado con cautela.

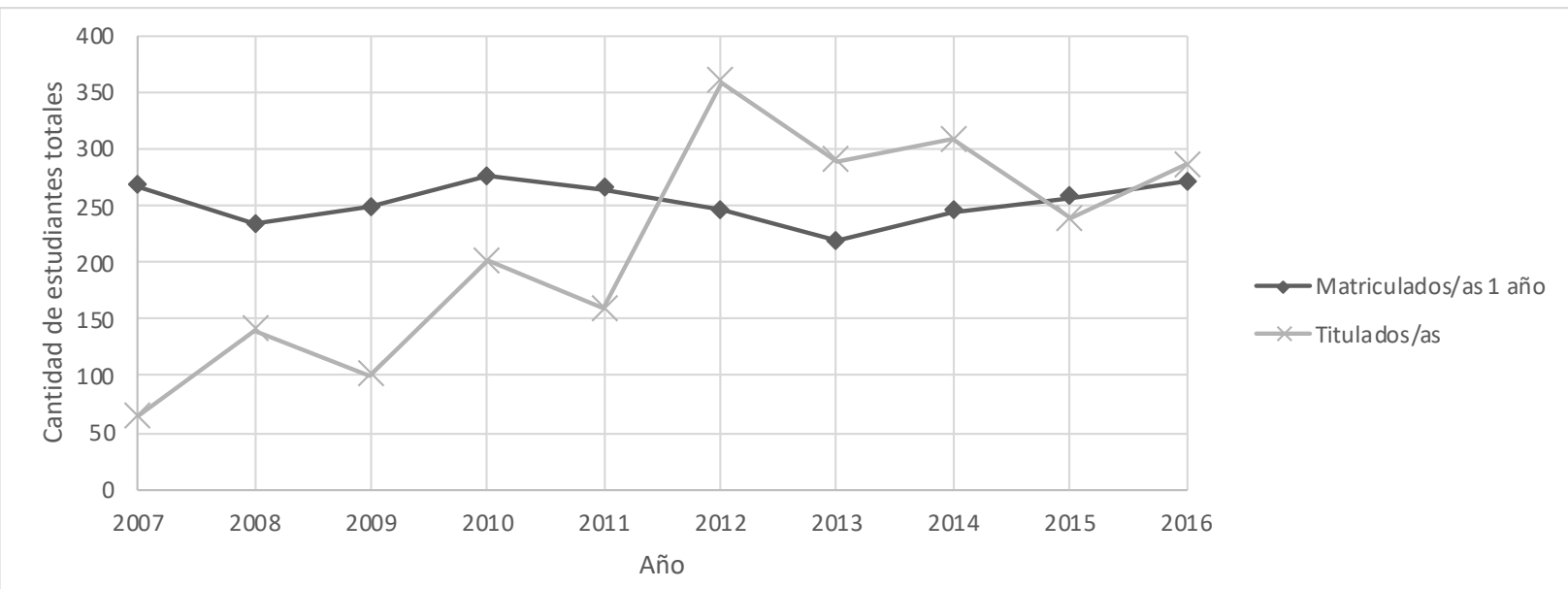

Fuente: Elaboración propia a partir de resultados.

Figura 9. Evolución histórica del número de estudiantes matriculados y titulados de Pedagogía en Física

La tendencia histórica muestra cómo aumenta el número de titulados sobre los matriculados en un mismo año, lo cual podría responder a efectos de la entrada en vigencia del decreto que reglamenta el ejercicio de la función docente (Decreto No. 352, 2003), el cual estipula la necesidad de contar con un título profesional para ejercer la docencia en Chile.

\subsection{Análisis cualitativo del perfil de egreso (Etapa 6)}

El perfil de egreso de las carreras refleja la identidad de cada programa formativo y declara cuáles son los conocimientos, habilidades, actitudes y valores que se compromete a desarrollar el futuro docente (Möller y Gómez, 2014). Para la formación de profesores en América Latina existe una diversidad de visiones respecto al perfil del egresado de algún programa de formación docente. Existe coincidencia en que un profesor recién egresado debe poseer amplio conocimiento de la disciplina a enseñar y de saberes pedagógicos, además de poseer cualidades como ser responsable, reflexivo, crítico y socialmente comprometido con su entorno (Rojas, 2013).

En la figura 10 se observa que el énfasis de los programas de formación de profesores de Física en Chile está en poseer conocimiento pedagógico sobre el disciplinar, y las habilidades de investigación -junto a las de liderazgo y trabajo en equipo-son escasamente consideradas. 


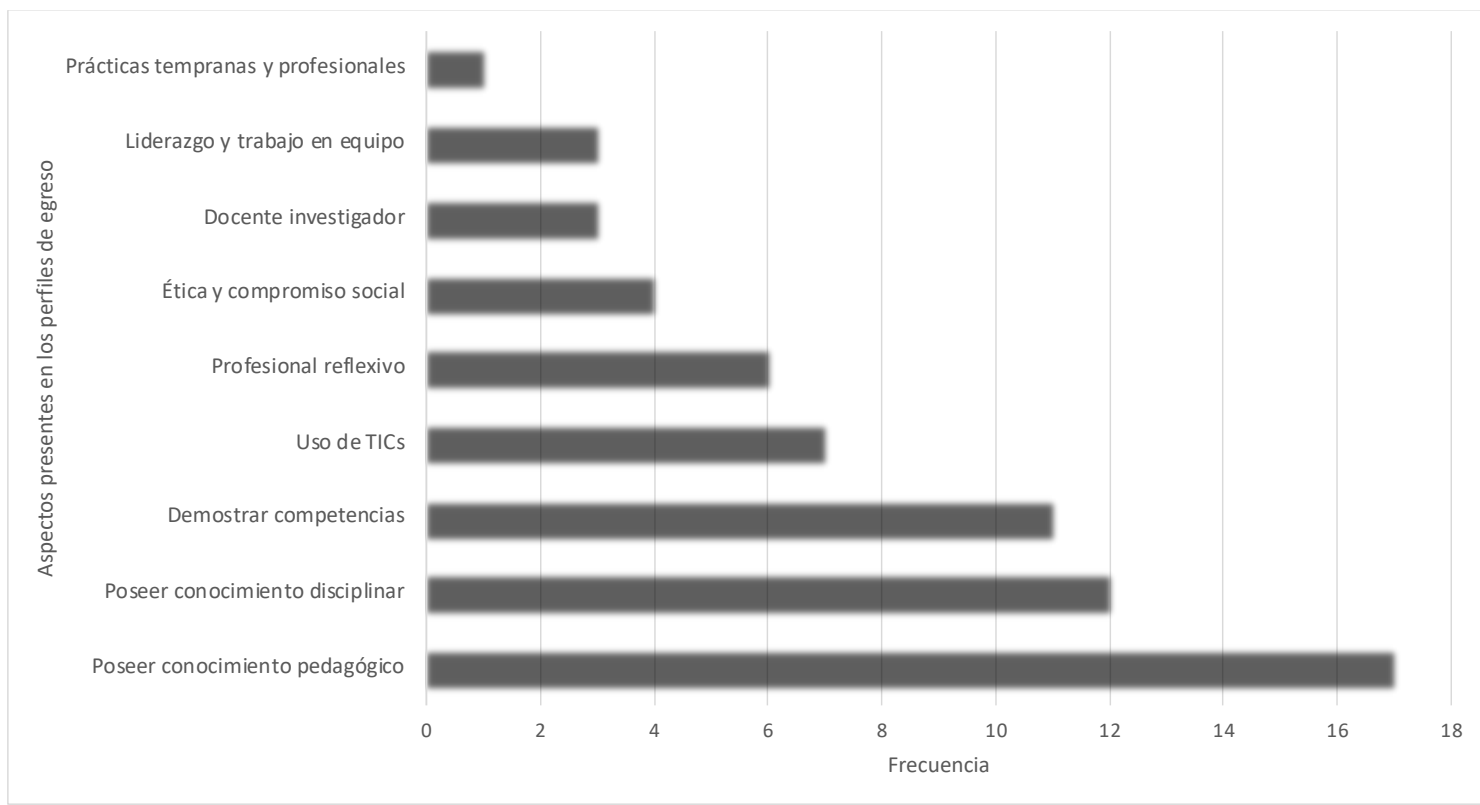

Figura 10. Características comunes en los perfiles de egreso analizados

\section{Conclusiones}

La promoción de reflexiones, estudios, debates y políticas público-privadas para fortalecer la FID en Chile ha constituido un desafío complejo y necesario de abordar para la promoción de una nueva ciudadanía. En este sentido, y dada la inexistencia de un documento de referencia con información actualizada y sistematizada sobre el panorama nacional de la formación de profesores en Física, se logró con este estudio cumplir el objetivo de identificar y describir las características de los actuales programas formativos de Pedagogía en Física de las universidades estatales y privadas del Consejo de Rectores de las Universidades Chilenas (CRUCH).

Los resultados obtenidos invitan a las IES, desde una perspectiva curricular, a reflexionar y evaluar el estado de la configuración actual de los programas formativos para desarrollar una oferta de formación continua que favorezca la profundización de algunas áreas que aparecen más débiles en la formación inicial, tales como: naturaleza de la ciencia, metodologías de investigación educativa y la propia didáctica específica.

Cabe resaltar también, que la evidencia de una débil articulación entre la formación pedagógica y de especialidad, coherente con resultados de estudios previos (Cisternas, 2011), invita de manera urgente a asumir responsable y propositivamente el desafío de generar redes de colaboración efectivas entre docentes de las diferentes áreas y facultades que participan de la formación docente.

Por otra parte, las diferencias por género detectadas en las tasas de matrícula para la carrera de Pedagogía en Física podrían atribuirse a que los resultados PSU en Ciencias y Matemática son globalmente mejores para hombres que para mujeres, lo que posibilita el acceso a la carrera para un género por sobre el otro (CONICYT, 2017). En diversos estudios se ha diagnosticado que el interés de hombres por carreras del área STEM (áreas de Ciencia, Tecnología, Ingeniería y Matemáticas, por sus siglas en inglés) suele ser mayor que para mujeres (Gelber et al., 2016) y, en este caso, la Pedagogía en Física podría no ser una excepción. No obstante, también hay estudios que indican una actitud mucho más favorable de las mujeres hacia la ciencia (Molina et al., 2013; Pelcastre et al., 2015), razón por la cual se requiere profundizar en nuevos estudios para entender qué ocurre con las decisiones vocacionales en el ingreso a carreras de pedagogía de disciplinas científicas. 
La particularidad de la formación de profesores en Chile, considerando la especificidad de la Física como disciplina, requiere de profundas reflexiones multidimensionales respecto a las necesidades de un sistema educativo que forme en y para una ciudadanía con conocimiento científico de base para transformar el mundo que le rodea. Sin embargo, las medidas adoptadas en últimos tiempos para regular la formación docente se han enfocado en nuevos procesos de acreditación obligatorios para las instituciones que imparten el programa, así como en un proceso de evaluación estandarizado orientado a establecer si el futuro docente posee o no las capacidades para ejercer labores educativas. En ese sentido, queda aún la necesidad y desafío de generar nuevas iniciativas que permitan mejorar la calidad de los programas de formación de profesores, atendiendo a las debilidades detectadas en este estudio y otros similares.

Por último, si bien el estudio realizado aporta evidencias generales sobre la actual situación de la formación de profesores de Física en Chile, surge la necesidad de profundizar en el análisis de los programas por institución, revisando a detalle su estructura y contenidos, en coherencia con sugerencias establecidas desde la OCDE (2017). No obstante, a pesar de lo incipiente del mismo, se considera un insumo pedagógico-didáctico relevante para el replanteamiento de directrices institucionales de los organismos estatales que son parte del Sistema de Aseguramiento de la Calidad de la Educación.

\section{Referencias}

Adaros, M. (2013). Implicancias de los nuevos estándares pedagógicos para el desarrollo de la competencia emocional en la formación inicial del profesorado. Temas de Educación, 19(1), 7-24.

https://revistas.userena.cl/index.php/teduacion/article/view/377

Adúriz-Bravo, A. (2005). Una introducción a la naturaleza de las ciencias: la epistemología en la enseñanza de las ciencias naturales. Fondo de Cultura Económica.

Agencia Calidad de la Educación (2014). Informe Nacional Resultados Chile Pisa 2012.

http://archivos.agenciaeducacion.cl/Informe Nacional Resultados Chile PISA 2012.pdf

Agencia Calidad de la Educación (2018). Resultados educativos 2017.

http://archivos.agenciaeducacion.cl/PPT Conferencia ER 2017 web 3.pdf

Arellano, M., Jara, R., Merino, C., Quintanilla, M. y Cuellar, L. (2008). Estudio comparativo de dos instrumentos de evaluación diagnóstica aplicados a profesores de Química en formación: un estudio piloto. Revista electrónica de Enseñanza de las Ciencias, 7(1), 1-22.

http://reec.uvigo.es/volumenes/volumen7/ART1 Vol7 N1.pdf

Atalar, F. B. y Ergun, M. (2018). Evaluation of the knowledge of science teachers with didactic transposition theory. Universal Journal of Educational Research, 6(1), 201-210.

http://doi.org/10.13189/ujer.2018.060130

Ávalos, B. (2003). La formación docente inicial en Chile. IESALC. Digital observatory for higher education in Latin America and the Caribbean.

http://www.oei.es/docentes/info pais/informe formacion docente chile iesalc.pdf

Bará, J., Córdoba, J. F., de Luis, R., Hernández, J. y Martín, P. (2002). Evaluación transversal del rendimiento académico de las ingenierías técnicas. Sociedad Anónima de Fotocomposición.

Barber, M. y Mourshed, M. (2007). Cómo hicieron los sistemas educativos con mejor desempeño del mundo para alcanzar sus objetivos. PREAL. http://eduteka.icesi.edu.co/articulos/InformeMcKensey

Barrón, C. (2015). Concepciones epistemológicas y práctica docente. Una revisión. Revista de Docencia Universitaria, 13(1), 35-56. https://doi.org/10.4995/redu.2015.6436 
Bolívar, A. (2005). Conocimiento didáctico del contenido y didácticas específicas. Profesorado. Revista de currículum y formación del profesorado, 9(2), 1-39.

https://recyt.fecyt.es/index.php/profesorado/article/view/42681

Cabezas, V. y Claro, F. (2011). Valoración social del profesor en Chile: ¿cómo atraer a alumnos talentosos a estudiar pedagogía? Temas de la Agenda Pública, 6(42), 1-16.

Carvalho, A. M. P. y Gil-Pérez, D. (2011). Formação de professores de ciências. Tendências e inovações [Formación de profesores de ciencias. Tendencias e innovaciones]. Cortez Editora.

Centro de Investigación Avanzada en Educación. (2017). Evolución de la matrícula en carreras de formación inicial docente (2005-2017). Observatorio Formación Docente/CIAE.

http://www.ciae.uchile.cl/index.php?page=view noticias\&lanqSite=es\&id=1207

Centro de Investigación Avanzada en Educación. (2018). Evolución de la oferta de formación docente en carreras de Pedagogía en Educación Media. Observatorio Formación Docente/CIAE.

http://ciae.uchile.cl/index.php?page=view noticias\&id=1345\&langSite=es

Centro de Perfeccionamiento, Experimentación e Investigaciones Pedagógicas. (2018). Resultados Nacionales, Evaluación Nacional Diagnóstica de la Formación Inicial Docente 2017. http://www.cpeip.cl/wpcontent/uploads/2018/07/Informe-Nacional-END-2017.pdf

Chevallard, Y. (2013). Sobre a teoria da transposição didática: algumas considerações introdutórias [Sobre la teoría de la transposición didáctica: algunas consideraciones introductorias]. Revista de Educação, Ciências e Matemática, 3(2). 1-14. http://publicacoes.unigranrio.edu.br/index.php/recm/article/view/2338

Cisternas, T. (2011). La investigación sobre formación docente en Chile. Territorios explorados e inexplorados. Revista Calidad en la Educación, (35), 131-164. http://dx.doi.org/10.31619/caledu.n35.98

Claro, F. (2003). Panorama docente de las ciencias naturales en Educación Media. Revista de Educación, (307), 13-21.

Claro, F., Paredes, R. D., Bennett, M. y Wilson, T. (2013). Incentivos para estudiar pedagogía: el caso de la beca vocación de profesor. Estudios públicos, (131), 37-59.

Cofré, H., Camacho, J., Galaz, A., Jiménez, J., Santibáñez, D. y Vergara, C. (2010). La educación científica en Chile: debilidades de la enseñanza y futuros desafíos de la educación de profesores de ciencia. Estudios pedagógicos, 36(2), 289-303. http://revistas.uach.cl/index.php/estped/article/view/3250

Comisión Nacional de Investigación Científica y Tecnología. (2017). Diagnóstico Igualdad de Género en Ciencia, Tecnología e Innovación en Chile. http://www.conicyt.cl/wp-content/uploads/2015/03/ Diagnostico-Equidad-de-Genero-en-CTI-MESA-CONICYT 2017.pdf

Consejo Nacional de Educación. (2017b). Índices 2017. Tendencias Matrícula Pregrado Educación Superior. https://www.cned.cl/sites/default/files/presentacion indices matricula 2017.pdf

Creswell, J. y Plano, V. (2011). Choosing a mixed methods design. En J. Creswell y V. Plano Clark (Eds.), Designing and conducting mixed methods research (pp. 53-106). Sage.

Cuellar, L. y Siso, Z. (2017). La naturaleza de la ciencia como ámbito de reflexión e inclusión en el aula para la promoción de competencias de pensamiento científico. Enseñanza de las Ciencias, (Extra), 3505-3510.

Decreto No. 352 (2003). Reglamenta ejercicio de la profesión docente. Ministerio de Educación, Santiago, Chile, 9 de octubre de 2003. http://bcn.cl/1v8ja 
Escobar, N. (2007). La práctica profesional docente desde la perspectiva de los estudiantes practicantes y tutores. Acción pedagógica, 16(1), 182-193. http://www.saber.ula.ve/handle/123456789/17286 
Fagúndez, T. y Castells, M. (2012). La argumentación en clases universitarias de física: una perspectiva retórica. Enseñanza de las ciencias. Revista de investigación y experiencias didácticas, 30(2), 153-174.

Gatti, B. (2015). Formação de professores: licenciaturas, currículos e políticas [Formación de profesores: licenciaturas, currículos y políticas]. Movimento-Revista de Educação, (2). 1-18.

https://periodicos.uff.br/revistamovimento/article/view/32545

Gelber, D., Treviño, E. y Inostroza, P. (2016). Inequidad de género en los logros de aprendizaje en educación primaria ¿Qué nos puede decir TERCE? Resumen ejecutivo. UNESCO.

https://unesdoc.unesco.org/ark:/48223/pf0000244348

Giroux, S. y Tremblay, G. (2004). Metodología de las ciencias humanas: la investigación en acción. Fondo de Cultura Económica.

Gómez-Martínez, Y., Carvalho, A. M. y Sasseron, L. H. (2014). Naturaleza de las ciencias en la enseñanza de la física. Algunas consideraciones relevantes y ejemplos en el aula. En M. Quintanilla, S. Daza y H. Cabrera (Eds.), Historia y Filosofía de las Ciencia. Aporte para una "nueva aula de ciencias", promotora de ciudadanía y valores (pp. 322-341). Bellaterra.

González, M. y González, R. M. (2008). Competencias genéricas y formación profesional: un análisis desde la docencia universitaria. Revista iberoamericana de educación, 47, 185-209.

https://rieoei.org/RIE/article/view/710

Harris, J., Mishra, P. y Koehler, M. (2009). Teachers' technological pedagogical content knowledge and learning activity types: curriculum-based technology integration reframed. Journal of Research on Technology in Education, 41(4), 393-416. https://doi.org/10.1080/15391523.2009.10782536

Hernández, C., López-Fernández, L., González-Donoso, A. y Tecpan-Flores, S. (2018). Impacto de estrategias de aprendizaje activo sobre el conocimiento disciplinar de futuros profesores de física, en un curso de didáctica. Pensamiento Educativo. Revista de Investigación Educacional Latinoamericana, 55(1), 112. http://doi.org/10.7764/PEL.55.1.2018.6

Hernández, R., Fernández, C., y Baptista, P. (2014). Metodología de la investigación (6a. ed.).Mc Graw Hill.

Justino, N. y de Almeida, P. (2016). A licenciatura em Física da UNESP de Presidente Prudente: uma análise de depoimentos de alunos sobre questões curriculares [El grado de Física en al UNESP: un análisis de los testimonios de los estudiantes sobre temas curriculares]. En R. Nardi, y B. S. C. Cortela (Eds.), Formação Inicial de Professores de Física em Universidades Pública. Editora Lavraria da Física.

Koponen, I. y Mantyla, T. (2006). Generative role of experiments in physics and in teaching physics: a suggestion for epistemological reconstruction. Science \& Education, 15, 31-54.

https://doi.org/10.1007/s11191-005-3199-6

López, Á. D., Flores, F. y Gallegos, L. (2000). La formación de docentes en física para el bachillerato. Reporte y reflexión sobre un caso. Revista Mexicana de Investigación Educativa, 5(9), 113-135.

Matthews, M. (2014). International handbook of research in history, philosophy and science teaching. Springer.

Maura, V. G. y Tirados, R. M. G. (2008). Competencias genéricas y formación profesional: un análisis desde la docencia universitaria. Revista iberoamericana de educación, 47, 185-209.

Ministerio de Educación. (2012). Estándares orientadores para carreras de pedagogía en educación media. LOM Ediciones. https://www.cpeip.cl/wp-content/uploads/2016/07/libromediafinal.pdf 
Molina, M., Carriazo, J., y Casas, J. (2013).Estudio transversal de las actitudes hacia la ciencia em estudiantes de grados quinto a undécimo. Adaptación y aplicación de un instrumento para valorar actitudes. TED, 33, 103-122.

Möller, I., y Gómez, H. (2014). Coherencia entre perfiles de egreso e instrumentos de evaluación en carreras de educación básica en Chile. Calidad en la Educación, (41), 17-49.

http://dx.doi.org/10.31619/caledu.n41.58

Montecinos, C., y Walker, H. (2005). Habilitación y certificación para los profesores en Chile. Calidad en la Educación, (23), 127-141. http://dx.doi.org/10.31619/caledu.n23.292

OCDE. (2016). PISA 2015 Results (Volume 1): Excellence and equity in education.

https://www.oecd.org/education/pisa-2015-results-volume-i-9789264266490-en.htm

OCDE. (2017). Education in Chile, Reviews of national policies for education.

https://www.oecd.org/education/education-in-chile-9789264284425-en.htm

Pelcastre, L., Gómez, A. R. y Zavala, G. (2015). Actitudes hacia la ciencia de estudiantes de educación preuniversitaria del centro de México. Revista Eureka sobre Enseñanza y Divulgación de las Ciencias, 12(3). 475-490. https://revistas.uca.es/index.php/eureka/article/view/2936

Perrenoud, P. (2005). Diez nuevas competencias para enseñar. Educatio Siglo XXI, (23), 223-229. https://revistas.um.es/educatio/article/view/127

Quintanilla, M. (2017). La historia de la ciencia en la investigación didáctica. Aporte a la formación y el desarrollo profesional del profesorado de ciencias. Bellaterra.

Quintanilla, M., Izquierdo, M. y Adúriz-Bravo, A. (2005). Avances en la construcción de marcos teóricos para incorporar la historia de la ciencia en la formación inicial del profesorado de Ciencias Naturales. Enseñanza de las Ciencias, (Extra) VII Congreso.

https://ddd.uab.cat/pub/edlc/edlc a2005nEXTRA/edlc a2005nEXTRAp478avacon.pdf

Roth, K. J. (2007). Science teachers as researchers. En S. K. Abell y N. G. Lederman (Eds.), Handbook of Research on Science Education (Vol. II) (pp. 1205-1259). Lawrence Erlbaum.

Ruay, R. (2018). El profesor como investigador de su acción pedagógica en la formación inicial docente. Revista Boletín Redipe, 7(11), 82-87. https://revista.redipe.org/index.php/1/article/view/636

Sasseron, L., Briccia, V. y de Carvalho, A. M. P. (2013). Aspectos da natureza das Ciências em sala de aula: exemplos do uso de textos científicos em prol do processo de Alfabetização Científica dos estudantes [Aspectos de la naturaleza de la Ciencia en el aula: ejemplos de uso de textos científicos a favor del proceso de alfabetización científica de los estudiantes]. En C. Celestino y M. Brzezinski (Eds.). Aprendendo ciência e sobre sua natureza: abordagens históricas e filosóficas (pp. 265-276). Tipographia Editora Expressa.

Servicio de Información de Educación Superior MINEDUC (2017). Bases de datos de matriculados en educación Superior 2007-2016. https://www.mifuturo.cl/bases-de-datos-de-matriculados/

Shulman, L. S. (2015). PCK: Its genesis and exodus. In A. Berry, P. Friedrichsen y J. Loughran (Eds). Reexamining pedagogical content knowledge in science education (pp. 13-23). Routledge.

Sotomayor, C. y Gysling, J. (2011). Estándares y regulación de calidad de la formación de profesores: discusión del caso chileno desde una perspectiva comparada. Calidad en la Educación, (35), 91-129.

Tejada, F. (2009). Competencias docentes. Revista de Currículum y Formación de Profesorado, 13(2), 1-15. https://recyt.fecyt.es/index.php/profesorado/article/view/42339 
UNESCO. (2012). Antecedentes y criterios para la elaboración de políticas docentes en América Latina y el Caribe. OREALc. https://unesdoc.unesco.org/ark:/48223/pf0000223249

Vaillant, D. (2013). Formación inicial del profesorado en América Latina: dilemas centrales y perspectivas. Revista Española de Educación Comparada, (22), 185-206. https://doi.org/10.5944/reec.22.2013.9329

Vogt, W. P. (2011). SAGE Quantitative Research Methods. Sage. 